\title{
FORMAL HODGE THEORY
}

\author{
LUCA BARBIERI-VIALE
}

\begin{abstract}
We introduce formal (mixed) Hodge structures (of level $\leq 1$ ) in such a way that the Hodge realization of Deligne's 1-motives extends to a realization from Laumon's 1-motives to formal Hodge structures (of level $\leq 1$ ) providing an equivalence of categories.
\end{abstract}

Let $\mathrm{MHS}_{1}^{\text {fr }}$ denote the category of torsion free graded polarizable mixed Hodge structures of level $\leq 1$. We have a nice algebraic description of this category via $\mathcal{M}_{1}^{\mathrm{fr}}$ the category of Deligne's 1-motives [5] ( $c f$. also [3], including torsion, one obtains 1motives with torsion describing $\mathrm{MHS}_{1}$ ). Actually, Deligne's Hodge realization provide an equivalence

$$
T_{\text {Hodge }}: \mathcal{M}_{1}^{\mathrm{fr}} \stackrel{\simeq}{\longrightarrow} \mathrm{MHS}_{1}^{\mathrm{fr}}
$$

such that Cartier duality on $\mathcal{M}_{1}^{\mathrm{fr}}$ is transformed in $\operatorname{Hom}(-, \mathbb{Z}(1))$ on $\mathrm{MHS}_{1}^{\mathrm{fr}}$. Moreover, we have a natural generalization of Deligne's 1-motives due to Laumon [6]. A Laumon 1-motive $M:=[F \stackrel{u}{\rightarrow} G]$ is a commutative formal group $F=F^{0} \times F_{\text {ét }}$, with torsion free étale part $F_{\text {ét }}$, a commutative connected algebraic group $G$ and a map of abelian fppf-sheaves $u: F \rightarrow G$. Let $\mathcal{M}_{1}^{a \text { fr }}$ denote the category of Laumon's 1motives and refer to its objects as 1-motives for short. Note that Cartier duality on $\mathcal{M}_{1}^{\text {fr }}$ canonically extends to $\mathcal{M}_{1}^{a \text {,fr }}$ (see [6]).

The purpose of this note is to introduce the abelian category $\mathrm{FHS}_{1}$ of formal mixed Hodge structures (of level $\leq 1$ ) in order to extend the Hodge realization $T_{\text {Hodge }}$ of Deligne's 1-motives $\mathcal{M}_{1}^{\text {fr }}$ to a realization $T_{\oint}$ from Laumon's 1-motives $\mathcal{M}_{1}^{a \text {,fr }}$ to $\mathrm{FHS}_{1}^{\mathrm{fr}} \subset \mathrm{FHS}_{1}$. We have that $\mathrm{MHS}_{1}^{\mathrm{fr}} \subset \mathrm{FHS}_{1}^{\mathrm{fr}}$ in a canonical way, i.e., there is a fully faithful embedding such that the natural involution (Cartier duality) on $\mathrm{MHS}_{1}^{\mathrm{fr}}$ extends to an involution on $\mathrm{FHS}_{1}^{\mathrm{fr}}$.

For the sake of exposition we here confine our study to level $\leq 1$ mixed Hodge structures. However, it is conceivable and suitable to consider formal mixed Hodge structures with arbitrary Hodge numbers: generalizing our definition below it's not that difficult (we will treat such a matter nextly, cf. [1,2.12] for the general setting). For example, enriched Hodge structures [4] (of level $\leq 1$ ) can easily be recovered as "special" formal Hodge structures (see also [2] for details). In [2] we are also providing a "sharp" De Rham realization generalizing Deligne's construction of De Rham realization in [5]. The main result of this paper can be summarized in the following way.

Received by the editors November 28, 2005. 
Theorem. There is an equivalence of categories with involution

$$
T_{\oint}: \mathcal{M}_{1}^{a, \mathrm{fr}} \stackrel{\simeq}{\longrightarrow} \mathrm{FHS}_{1}^{\mathrm{fr}}
$$

between Laumon's 1-motives and torsion free formal Hodge structures (of level $\leq 1$ ) providing a diagram

$$
\begin{array}{cccc}
\mathcal{M}_{1}^{\mathrm{fr}} & \stackrel{\simeq}{\rightrightarrows} & \mathrm{MHS}_{1}^{\mathrm{fr}} \\
\uparrow \downarrow & & \uparrow \downarrow \\
\mathcal{M}_{1}^{a, \mathrm{fr}} & \stackrel{\cong}{ } & \mathrm{FHS}_{1}^{\mathrm{fr}}
\end{array}
$$

where

- $\mathcal{M}_{1}^{\mathrm{fr}} \hookrightarrow \mathcal{M}_{1}^{a \text { fr }}$ and $\mathrm{MHS}_{1}^{\mathrm{fr}} \hookrightarrow \mathrm{FHS}_{1}^{\mathrm{fr}}$ are canonical inclusions,

- $\mathcal{M}_{1}^{a \text { fr }} \rightarrow \mathcal{M}_{1}^{\mathrm{fr}}$ and $\mathrm{FHS}_{1}^{\mathrm{fr}} \rightarrow \mathrm{MHS}_{1}^{\mathrm{fr}}$ are "forgetful functors" denoted ( ) $\rightsquigarrow$ ( )ét, which are left inverses of the inclusions,

- $T_{\oint}(M)$ coincide with $T_{\text {Hodge }}(M)$ if $M=M_{\text {ét }}$ and, in general, we have a formula

$$
T_{\oint}(M)_{\text {ét }}=T_{\text {Hodge }}\left(M_{\text {ét }}\right) \text {. }
$$

The plan of the paper is the following. In Section 1 we introduce the category $\mathrm{FHS}_{1}$. In Section 2 we construct $T_{\oint}$ proving the theorem.

\section{Formal Hodge Structures}

1.1. Paradigma. Consider a commutative formal group $H=H^{0} \times H_{\mathbb{Z}}$ over $\mathbb{C}$ along with a mixed Hodge structure on the étale part $H_{\mathbb{Z}}$, i.e., say $H_{\text {ét }}:=\left(H_{\mathbb{Z}}, W_{*}, F_{\text {Hodge }}^{*}\right) \in$ $\mathrm{MHS}_{1}$ for short. For the mixed Hodge structure $H_{\text {ét }} \in \mathrm{MHS}_{1}$ we here denote $H_{\mathbb{Z}}$ the finitely generated abelian underlying group, along with the weight filtration $W_{-2} \subseteq$ $W_{-1}$ of $H_{\mathbb{Q}}:=H_{\mathbb{Z}} \otimes \mathbb{Q}$ and $F_{H o d g e}^{0} \subseteq H_{\mathbb{C}}:=H_{\mathbb{Z}} \otimes \mathbb{C}$ the Hodge filtration. We say that $H$ is free if the étale part of the formal group is free, so that: $H_{\mathbb{Z}}=\mathbb{Z}^{r}$ and $H^{0}=\widehat{\mathbb{C}}^{s}$ non-canonically. (Note that here $\widehat{\mathbb{C}}$ denotes the connected formal additive group). For $H$ free we also denote by $W_{*} H_{\text {ét }}$ and $\operatorname{gr}_{*}^{W} H_{\text {ét }}$ the corresponding objects of $\mathrm{MHS}_{1}$.

1.1.1. Definition. Define a formal Hodge structure (of level $\leq 1$ ) as follows: (i) a formal group $H$ such that $H_{\text {ét }} \in \mathrm{MHS}_{1}$, (ii) a finite dimensional $\mathbb{C}$-vector space $V$ with a two steps filtration $V^{0} \subseteq V^{1} \subseteq V$ by sub-spaces, (iii) a group homomorphism $v: H \rightarrow V$ and (iv) a $\mathbb{C}$-isomorphism $\sigma: H_{\mathbb{C}} / F_{\text {Hodge }}^{0} \stackrel{\simeq}{\rightarrow} V / V^{0}$ restricting to an isomorphism $W_{-2} H_{\mathbb{C}} \cong V^{1} / V^{0}$. We further assume that the following condition holds: if $v_{\mathbb{Z}}: H_{\mathbb{Z}} \rightarrow V$ is the induced map, $c: H_{\mathbb{Z}} \rightarrow H_{\mathbb{C}} / F_{\text {Hodge }}^{0}$ is the canonical map and pr: $V \rightarrow V / V^{0}$ is the projection then the following

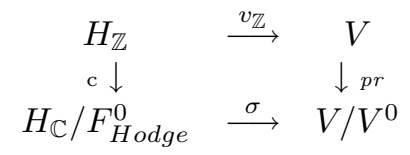

commutes. Denote $(H, V)$ for short such a structure. 
Define a morphism $\phi$ between $(H, V)$ and $\left(H^{\prime}, V^{\prime}\right)$ as follows. We let $\phi:=(f, g)$ be a pair of maps in the following commutative square

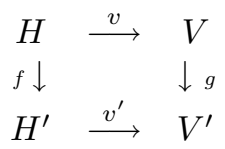

where $f: H \rightarrow H^{\prime}$ is a homomorphism of formal groups such that $f_{\text {ét }}: H_{\text {ét }} \rightarrow H_{\text {ét }}^{\prime}$ is a map in $\mathrm{MHS}_{1}$ and $g: V \rightarrow V^{\prime}$ is a $\mathbb{C}$-homomorphism compatible with the filtrations, i.e., $g\left(V^{i}\right) \subseteq V^{i}$ for $i=0,1$. We further assume that the following diagram commutes

$$
\begin{array}{ccc}
H_{\mathbb{C}} / F_{\text {Hodge }}^{0} & \stackrel{\sigma}{\longrightarrow} & V / V^{0} \\
\bar{f} \downarrow & & \downarrow \bar{g} \\
H_{\mathbb{C}}^{\prime} / F_{\text {Hodge }}^{0} & \stackrel{\sigma^{\prime}}{\longrightarrow} & V^{\prime} / V^{\prime 0}
\end{array}
$$

where $\bar{f}$ and $\bar{g}$ are the canonically induced maps.

1.1.2. Definition. Let $\mathrm{FHS}_{1}$ denote the category whose objects are $(H, V)$, the morphisms are $\phi=(f, g)$ as above and the composition is given by gluing the squares (2) (the condition (3) is preserved by gluing). Let $\mathrm{FHS}_{1}^{\mathrm{fr}} \subset \mathrm{FHS}_{1}$ denote the full subcategory given by $(H, V)$ such that $H$ is free.

1.1.3. Proposition. The category $\mathrm{FHS}_{1}$ is abelian. A short exact sequence

$$
0 \rightarrow(H, V) \rightarrow\left(H^{\prime}, V^{\prime}\right) \rightarrow\left(H^{\prime \prime}, V^{\prime \prime}\right) \rightarrow 0
$$

is given by an exact sequence on each component (formal groups and filtered vector spaces) so that

$$
0 \rightarrow H_{\text {ét }} \rightarrow H_{\text {ét }}^{\prime} \rightarrow H_{\text {ét }}^{\prime \prime} \rightarrow 0
$$

is exact in $\mathrm{MHS}_{1}$.

Proof. Straightforward.

1.2. Étale structures. We can recover mixed Hodge structures as follows.

1.2.1. Definition. Define $(H, V)_{\text {ét }}:=\left(H_{\mathbb{Z}}, V / V^{0}\right)$ where $\left(H_{\mathbb{Z}}\right)_{\text {ét }}:=H_{\text {ét }}, v_{\text {ét }}: H_{\mathbb{Z}} \rightarrow$ $V / V^{0}$ is the composition of pr and $v_{\mathbb{Z}}(c f .1 .1 .1)$ and

$$
\left(V / V^{0}\right)^{0}:=0 \subseteq\left(V / V^{0}\right)^{1}:=V^{1} / V^{0} \subseteq V / V^{0} .
$$

Say that a formal Hodge structure is étale if $(H, V)=(H, V)$ ét, i.e., if $H^{0}=V^{0}=0$.

Given $\left(H_{\mathbb{Z}}, W_{*}, F_{H o d g e}^{*}\right) \in \mathrm{MHS}_{1}$ there is a natural way to provide an étale one as follows. Set $H:=H_{\mathbb{Z}}, H_{\text {ét }}:=\left(H_{\mathbb{Z}}, W_{*}, F_{\text {Hodge }}^{*}\right), H^{0}=0, V:=H_{\mathbb{C}} / F_{\text {Hodge }}^{0}, V^{0}:=0$, $V^{1}:=W_{-2} H_{\mathbb{C}}, \sigma$ is the identity and the map $v:=c$ is induced by the canonical map $t: H_{\mathbb{Z}} \rightarrow H_{\mathbb{C}}$. Denote

$$
c\left(H_{\mathbb{Z}}, W_{*}, F_{\text {Hodge }}^{*}\right):=\left(H_{\mathbb{Z}}, H_{\mathbb{C}} / F_{\text {Hodge }}^{0}\right)
$$

the canonical étale formal Hodge structure associated to a mixed Hodge structure, providing a functor $c: \mathrm{MHS}_{1} \rightarrow \mathrm{FHS}_{1}$. 
1.2.2. Lemma. The full subcategory $\mathrm{FHS}_{1}^{\text {ét }}$ of étale structures is equivalent to $\mathrm{MHS}_{1}$

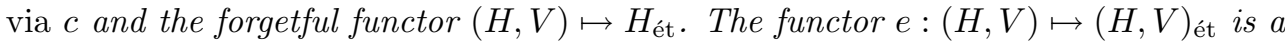
left inverse of the inclusion $\mathrm{FHS}_{1}^{\text {ét }} \subset \mathrm{FHS}_{1}$ and, for $\left(H^{\prime}, V^{\prime}\right) \in \mathrm{FHS}_{1}^{\text {ét }}$, we have

$$
\operatorname{Hom}\left((H, V),\left(H^{\prime}, V^{\prime}\right)\right) \subseteq \operatorname{Hom}\left((H, V)_{\text {ét }},\left(H^{\prime}, V^{\prime}\right)\right)
$$

where the equality holds if $v\left(H^{0}\right) \subseteq V^{0}$ (cf. 1.3.1 below).

Proof. Actually, for the equivalence, we are easily left to show that if $(H, V)$ is étale then $c\left(H_{\text {ét }}\right):=\left(H_{\mathbb{Z}}, H_{\mathbb{C}} / F_{H o d g e}^{0}\right) \cong(H, V)$. The claimed isomorphism is $(1, \sigma)$ granted by (1) since $V^{0}=H^{0}=0$.

For the other claims, let $(H, V) \in \mathrm{FHS}_{1}$ and $\left(H^{\prime}, V^{\prime}\right) \in \mathrm{FHS}_{1}^{\text {ét }}$ and consider a map $\phi=(f, g):(H, V) \rightarrow\left(H^{\prime}, V^{\prime}\right)$ whence induced maps $\bar{f}$ and $\bar{g}$ and a diagram

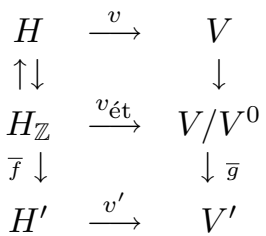

In fact $H^{\prime}=H_{\text {ét }}^{\prime}$ is étale thus $f\left(H^{0}\right)=0$ and $f$ factors through $H_{\mathbb{Z}}$ yielding $\bar{f}$ and, similarly, we get a filtered map $\bar{g}: V / V^{0} \rightarrow V^{\prime}$ since $V^{\prime 0}=0$ and $g\left(V^{0}\right)=0$. Now $\bar{\phi}:=(\bar{f}, \bar{g})$ yields a map by diagram chase. Note that if $v\left(H^{0}\right) \subseteq V^{0}$ then $(H, V) \rightarrow(H, V)_{\text {ét }}(c f .(5))$ and we can lift back, by composition, any morphism $\phi^{\prime}:(H, V)_{\text {ét }} \rightarrow\left(H^{\prime}, V^{\prime}\right)$ as the condition (3) is tautological.

1.2.3. Remark. Note that under the equivalence we then get a canonical inclusion $c: \mathrm{MHS}_{1}^{\mathrm{fr}} \hookrightarrow \mathrm{FHS}_{1}^{\mathrm{fr}}$ such that $e: \mathrm{FHS}_{1}^{\mathrm{fr}} \rightarrow \mathrm{MHS}_{1}^{\mathrm{fr}}$ is a left inverse and for $H^{\prime} \in \mathrm{MHS}_{1}^{\mathrm{fr}}$

$$
\operatorname{Hom}_{\mathrm{FHS}_{1}^{\mathrm{fr}}}\left((H, V),\left(H_{\mathbb{Z}}^{\prime}, H_{\mathbb{C}}^{\prime} / F_{H o d g e}^{0}\right)\right) \subseteq \operatorname{Hom}_{\mathrm{MHS}_{1}^{\mathrm{fr}}}\left(H_{\text {ét }}, H^{\prime}\right)
$$

1.3. Connnected structures. A $\mathbb{C}$-vector space $V$ will be regarded as an object $(0, V)$ of $\mathrm{FHS}_{1}$ filtered as $V=V^{1}=V^{0}$. Similarly, a formal group $H$ is regarded as an object $(H, 0)$ of $\mathrm{FHS}_{1}$ so that $H=H^{0} \times H_{\mathbb{Z}}$ and $H_{\mathbb{Z}}$ is pure of weight zero.

For $(H, V) \in \mathrm{FHS}_{1}$ we have that $V^{0}$ is a substructure of $(H, V)$ and we can consider the quotient $(H, V) / V^{0}=\left(H, V / V^{0}\right)$ in $\mathrm{FHS}_{1}$. We can also regard $(H, V)$ ét as a substructure of $(H, V) / V^{0}$ and we obtain a canonical exact sequence

$$
0 \rightarrow(H, V)_{\text {ét }} \rightarrow(H, V) / V^{0} \rightarrow H^{0} \rightarrow 0
$$

1.3.1. Definition. Say that $(H, V) \in \mathrm{FHS}_{1}$ is connected if $H=H^{0}$ is connected, i.e., if $(H, V)_{\text {ét }}=0$. Denote $\pi(H, V):=\left(H^{0}, V\right)$ the connected structure given by $V=V^{1}=V^{0}$ and the restriction of $v$ to $H^{0} \subseteq H$. Let $\mathrm{FHS}_{1}^{0}$ denote the full subcategory of $\mathrm{FHS}_{1}$ determined by connected structures.

Say that $(H, V) \in \mathrm{FHS}_{1}$ is special if $v\left(H^{0}\right) \subseteq V^{0}$, i.e., if $v: H \rightarrow V$ restricts to $v^{0}: H^{0} \rightarrow V^{0}$. Denote $\mathrm{FHS}_{1}^{s}$ the full subcategory of special structures and $(H, V)^{0}:=\left(H^{0}, V^{0}\right) \in \mathrm{FHS}_{1}^{0}$ the connected structure determined by $(H, V) \in \mathrm{FHS}_{1}^{s}$.

1.3.2. Lemma. The functor $(H, V) \mapsto \pi(H, V)$ is a left inverse of the inclusion $\iota: \mathrm{FHS}_{1}^{0} \subset \mathrm{FHS}_{1}$. The category $\mathrm{FHS}_{1}^{0}$ is equivalent to the category of linear mappings between finite dimensional $\mathbb{C}$-vector spaces. For $\left(H^{\prime}, V^{\prime}\right) \in \mathrm{FHS}_{1}^{0}$ and $(H, V) \in \mathrm{FHS}_{1}^{s}$

$$
\operatorname{Hom}\left(\left(H^{\prime}, V^{\prime}\right),(H, V)\right) \cong \operatorname{Hom}\left(\left(H^{\prime}, V^{\prime}\right),(H, V)^{0}\right)
$$


Proof. The first claim is clear. Moreover, the equivalence is provided by $(H, V) \mapsto$ Lie $(H) \rightarrow V$. Finally, a map from $\left(H^{\prime}, V^{\prime}\right)$ connected to $(H, V)$ special is given by a commutative square

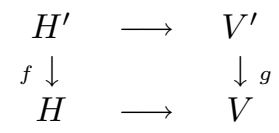

such that $f\left(H^{\prime}\right) \subseteq H^{0}$ and $g\left(V^{\prime}\right) \subseteq V^{0}$.

1.3.3. Remark. Note that $(H, V)$ with $H_{\text {ét }}$ pure of weight zero exists if and only if $V=V^{1}=V^{0}$. Thus if $(H, V)$ is special then $(H, V)^{0}$ is the largest connected formal substructure of $(H, V)$ and we have a non canonical extension

$$
0 \rightarrow\left(H^{0}, V^{0}\right) \rightarrow(H, V) \rightarrow(H, V)_{\text {ét }} \rightarrow 0
$$

From lemmas 1.2.2 and 1.3.2 it follows that the functors $(H, V) \mapsto(H, V)^{0}$ and $(H, V) \mapsto(H, V)_{\text {ét }}$ are, respectively, a right adjoint of $\mathrm{FHS}_{1}^{0} \subset \mathrm{FHS}_{1}^{s}$ and a left adjoint of $\mathrm{FHS}_{1}^{\text {ét }} \subset \mathrm{FHS}_{1}^{s}$. However, special structures do have disadvantages, see 2.2.5 and 2.3.2.

1.3.4. Proposition. The category $\mathrm{FHS}_{1}^{0}$ forms a Serre abelian subcategory of $\mathrm{FHS}_{1}$ yielding the extension

$$
0 \rightarrow \mathrm{FHS}_{1}^{0} \stackrel{\iota}{\rightarrow} \mathrm{FHS}_{1} \stackrel{e}{\rightarrow} \mathrm{MHS}_{1} \rightarrow 0
$$

where $\pi \iota=1$ and $e c=1$.

Proof. It follows from the lemmas 1.2.2, 1.3.2 and (4). In fact, it is clear (cf. 1.1.3) that $\mathrm{FHS}_{1}^{0}$ forms a Serre subcategory. Since $e\left(\mathrm{FHS}_{1}^{0}\right)=0$ we have a factorisation $\bar{e}: \mathrm{FHS}_{1} / \mathrm{FHS}_{1}^{0} \rightarrow \mathrm{MHS}_{1}$ via the canonical projection $t: \mathrm{FHS}_{1} \rightarrow \mathrm{FHS}_{1} / \mathrm{FHS}_{1}^{0}$ and

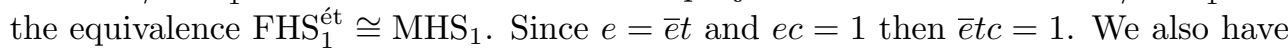
$t c \bar{e} \cong 1$ since applying $t$ to (4) for $(H, V) \in \mathrm{FHS}_{1}$ we get a natural isomorphism

$$
t c\left(H_{\text {ét }}\right) \cong t(H, V)_{\text {ét }} \cong t(H, V)
$$

1.4. Construction. We provide a Laumon 1-motive out of a free formal mixed Hodge structure (of level $\leq 1$ ). The construction is similar to [5, p. 55-56].

For $(H, V) \in \mathrm{FHS}_{1}^{\text {fr }}$ the Laumon 1-motive $\overrightarrow{(H, V)}:=[F \stackrel{u}{\rightarrow} G]$ functorially associated to $(H, V)$ is given as follows. Set $F:=H^{0} \times \operatorname{gr}_{0}^{W}\left(H_{\mathbb{Z}}\right)$. Since (1) holds true $W_{-1}\left(H_{\mathbb{Z}}\right)$ injects in $V$ via $v_{\mathbb{Z}}: H_{\mathbb{Z}} \rightarrow V$ in such a way that $W_{-1}\left(H_{\mathbb{Z}}\right) \cap V^{0}=0$. Set $G(\mathbb{C}):=V / W_{-1}\left(H_{\mathbb{Z}}\right)$ obtaining a diagram

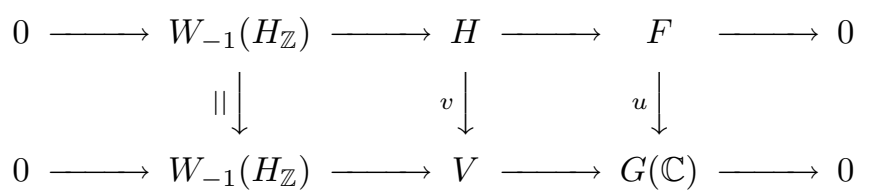


where $u$ is just induced by $v$. Regarding the complex group $G(\mathbb{C})$ we then have it in a diagram

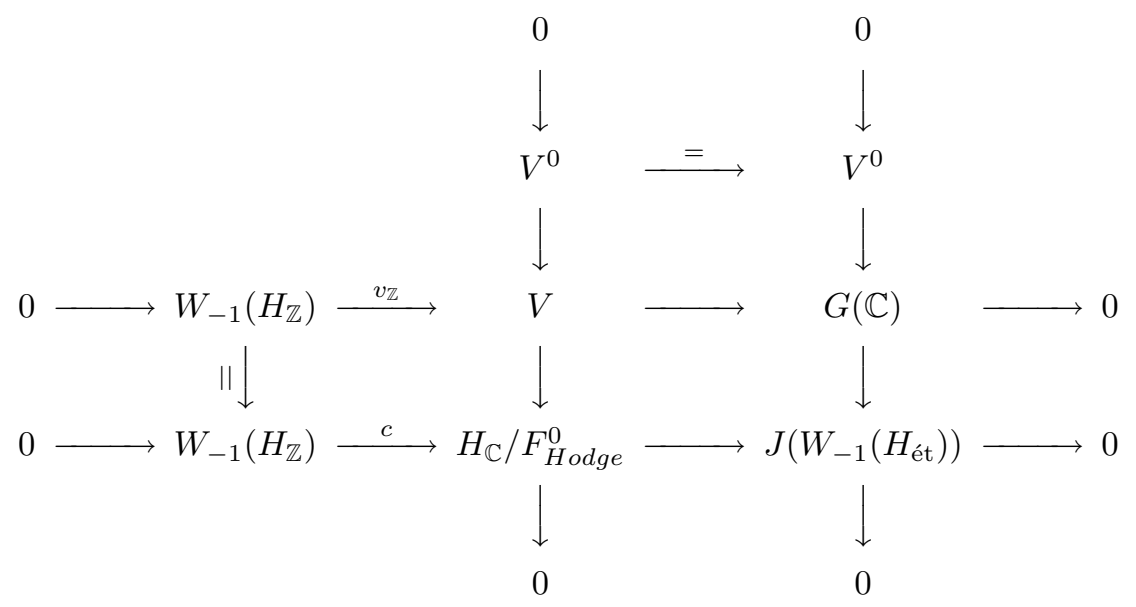

obtained via $\sigma$ and (1). This is showing that $G(\mathbb{C})$ is an extension of the complex torus $J\left(W_{-1}\left(H_{\text {ét }}\right)\right)$ by a $\mathbb{C}$-vector group. Thus, by G.A.G.A., we get the algebraic group $G$.

\section{Formal Hodge realization}

2.1. Paradigma. For a Laumon 1-motive $M=[F \stackrel{u}{\rightarrow} G] \in \mathcal{M}_{1}^{a, \text { fr }}$ over a field $k$ (algebraically closed of characteristic zero) we here denote $F=F^{0} \times F_{\text {ét }}$ the formal group where $F_{\text {ét }}$ is further assumed torsion free. Denote $V(G):=\mathbb{G}_{a}^{n} \subseteq G$ the additive factor and display the connected algebraic group $G$ as an extension

$$
0 \rightarrow V(G) \rightarrow G \rightarrow G_{\times} \rightarrow 0
$$

where $G_{\times}$is the semi-abelian quotient. The algebraic group $G_{\times}$is an extension of an abelian variety $A$ by a torus $T$.

2.1.1. Definition. For $M=[F \stackrel{u}{\rightarrow} G] \in \mathcal{M}_{1}^{a, \text { fr }}$ set $M_{\text {ét }}:=\left[F_{\text {ét }} \stackrel{u_{\text {ét }}^{\rightarrow}}{\rightarrow} G_{\times}\right] \in \mathcal{M}_{1}^{\text {fr }}$. Say that $M$ is étale if $M=M_{\text {ét }}$, i.e., it is a Deligne 1-motive. Say that $M$ is connected if $M_{\text {ét }}=0$, i.e., $F=F^{0}$ is connected and $G=V(G)$ is a vector group. Say that $M$ is special if $u\left(F^{0}\right) \subseteq V(G)$ and set $M^{0}:=\left[F^{0} \rightarrow V(G)\right]$.

2.1.2. Lemma. The functor $M \mapsto M_{\text {ét }}$ is a left inverse of the inclusion $\mathcal{M}_{1}^{\mathrm{fr}} \subset \mathcal{M}_{1}^{a, \mathrm{fr}}$ of Deligne's 1-motives and for $M^{\prime} \in \mathcal{M}_{1}^{\mathrm{fr}}$ we have

$$
\operatorname{Hom}\left(M, M^{\prime}\right) \subseteq \operatorname{Hom}\left(M_{\text {ét }}, M^{\prime}\right)
$$

If $M$ is special we then get an extension

$$
0 \rightarrow M^{0} \rightarrow M \rightarrow M_{\text {ét }} \rightarrow 0
$$

such that if $M^{\prime}$ is étale then $\operatorname{Hom}\left(M_{\text {ét }}, M^{\prime}\right) \cong \operatorname{Hom}\left(M, M^{\prime}\right)$ and if $M^{\prime}$ is connected then $\operatorname{Hom}\left(M^{\prime}, M^{0}\right) \cong \operatorname{Hom}\left(M^{\prime}, M\right)$. 
Proof. Let $M=[F \stackrel{u}{\rightarrow} G] \in \mathcal{M}_{1}^{a, \text { fr }}, M^{\prime}=\left[F^{\prime} \stackrel{u^{\prime}}{\rightarrow} G^{\prime}\right] \in \mathcal{M}_{1}^{\mathrm{fr}}$. Let $(f, g): M \rightarrow M^{\prime}$ be a map. Then get a diagram (cf. the proof of 1.2.2)

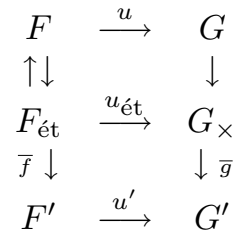

where $\bar{f}$ and $\bar{g}$ are the induced maps since $M^{\prime}$ is étale, yielding a map $(\bar{f}, \bar{g}): M_{\text {ét }} \rightarrow$ $M^{\prime}$. In fact, $\operatorname{Hom}\left(F, F^{\prime}\right)=\operatorname{Hom}\left(F_{\text {ét }}, F^{\prime}\right)$ because $F^{\prime}$ is étale and $F^{0}$ is mapped to zero and $\operatorname{Hom}\left(G, G^{\prime}\right)=\operatorname{Hom}\left(G_{\times}, G^{\prime}\right)$ because $\operatorname{Hom}\left(\mathbb{G}_{a}, \mathbb{G}_{m}\right)=\operatorname{Hom}\left(\mathbb{G}_{a}, A\right)=0$ and $G^{\prime}$ is semi-abelian. Moreover, $M \rightarrow M_{\text {ét }}$ if $M$ is special, yielding (8). For the isomorphisms then note that $\operatorname{Hom}\left(M^{0}, M^{\prime}\right)=0$ if $M^{\prime}$ is étale and, equivalently, $\operatorname{Hom}\left(M^{\prime}, M_{\text {ét }}\right)=0$ if $M^{\prime}$ is connected.

In general, we can regard $M_{\text {ét }}$ as a sub-1-motive of $M / V(G)$ and we obtain ( $c f$. (4)) a canonical exact sequence

$$
0 \rightarrow M_{\text {ét }} \rightarrow M / V(G) \rightarrow F^{0}[1] \rightarrow 0
$$

Denote $M_{\text {ét }}^{\natural}=\left[F_{\text {ét }} \stackrel{u^{\natural}}{\rightarrow} G^{\natural}\right] \in \mathcal{M}_{1}^{a, \text { fr }}(c f .[5])$ the universal $\mathbb{G}_{a}$-extension of $M_{\text {ét }}$. The algebraic group $G^{\natural}$ can be represented by an extension

$$
0 \rightarrow \operatorname{Ext}\left(M_{\text {ét }}, \mathbb{G}_{a}\right)^{\vee} \rightarrow G^{\natural} \rightarrow G_{\times} \rightarrow 0
$$

where $\operatorname{Ext}\left(M_{\text {ét }}, \mathbb{G}_{a}\right)^{\vee}$ is given by the dual vector space of $\mathbb{G}_{a}$-extensions of $M_{\text {ét }}$. The map $u^{\natural}: F_{\text {ét }} \rightarrow G^{\natural}$ is a canonical lifting of $u_{\text {ét }}: F_{\text {ét }} \rightarrow G_{\times}$.

Set $k=\mathbb{C}$. Recall that Deligne's Hodge realization (see [5])

$$
T_{\text {Hodge }}\left(M_{\text {ét }}\right):=\left(H_{\mathbb{Z}}, W_{*}, F_{\text {Hodge }}^{0}\right)
$$

of $M_{\text {ét }}$ is given by the pull-back

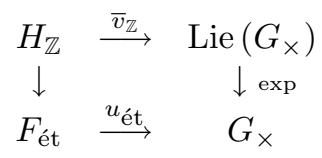

Here $W_{-1}:=H_{1}\left(G_{\times}\right), W_{-2}:=H_{1}(T)$ and

$$
F_{\text {Hodge }}^{0}:=\operatorname{Ker}\left(H_{\mathbb{C}} \rightarrow \operatorname{Lie}\left(G_{\times}\right)\right)
$$

2.1.3. Lemma. $([5,10.1])$ For $k=\mathbb{C}$ we have an isomorphism

$$
M_{\text {ét }}^{\natural} \cong\left[H_{\mathbb{Z}} / W_{-1} \stackrel{\bar{t}}{\rightarrow} H_{\mathbb{C}} / W_{-1}\right]
$$

here $\bar{t}$ is the induced map $t: H_{\mathbb{Z}} \rightarrow H_{\mathbb{C}} \bmod W_{-1}\left(H_{\mathbb{Z}}\right)$.

Actually (see $[5,10.1 .8]$ ) we have a bifiltered isomorphism (i.e., "periods")

$$
\tau: \operatorname{Lie}\left(G^{\natural}\right) \stackrel{\simeq}{\rightrightarrows} H_{\mathbb{C}}
$$


such that

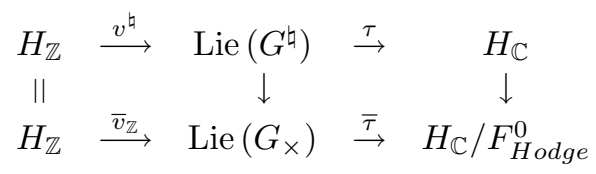

commutes. Here $t=\tau v^{\natural}$ where $v^{\natural}$ is the canonical map induced by $u^{\natural}$, $\operatorname{Lie}\left(G^{\natural}\right)$ is the pullback of $(10)$ along $\exp , H_{1}\left(G^{\natural}\right) \cong H_{1}\left(G_{\times}\right)=W_{-1}\left(H_{\mathbb{Z}}\right)$ and $\operatorname{Ext}\left(M_{\text {ét }}, \mathbb{G}_{a}\right)^{\vee} \cong$ $F_{\text {Hodge }}^{0}$.

2.1.4. Example. (cf. $[1,1.1 \& 3.3])$ For $X$ proper over a field $k$, char $(k)=0$, set $G:=\operatorname{Pic}_{X / k}^{0}$ and let $M=[0 \rightarrow G]$ be the corresponding 1-motive. Here $G_{\times} \cong \operatorname{Pic}_{X . / k}^{0}$ and $G^{\natural} \cong \mathbb{P i c}_{X}^{\natural, / k}$ are given by simplicial Pic and $\downarrow-$ Pic functors of a smooth proper hypercovering $X$. of $X$. Thus $H_{\mathbb{Z}}=H^{1}\left(X_{\text {an }}, \mathbb{Z}\right)$, Lie $\left(G^{\natural}\right)=H_{D R}^{1}(X)$ and $\tau: H_{D R}^{1}(X) \cong H^{1}\left(X_{\text {an }}, \mathbb{C}\right)$ by cohomological descent over $k=\mathbb{C}$.

2.2. Construction. Extending Deligne's Hodge realization for a given Laumon 1motive $M=[F \stackrel{u}{\rightarrow} G]$ over $\mathbb{C}$ consider the pull-back $T_{\S}(F)$ of $u: F \rightarrow G$ along $\exp :$ Lie $(G) \rightarrow G$, i.e., it fits in the following diagram

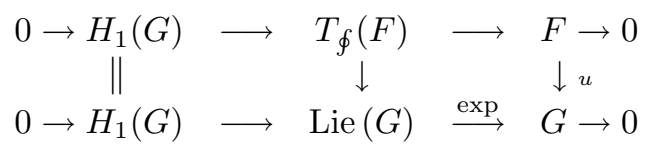

Here $T_{\oint}(F)$ is a formal group and we get a natural group homomorphism $v: T_{\oint}(F) \rightarrow$ Lie $(G)$. We are going to show that

$$
T_{\oint}(M):=\left(T_{\oint}(F), \operatorname{Lie}(G)\right) \in \mathrm{FHS}_{1}^{\mathrm{fr}}
$$

is a formal Hodge structure. Note that if $M$ is connected then $T_{\oint}(M)=M$.

2.2.1. Remark. The additional data coming from Lie is really needed if we allow additive factors! For example, let $W \rightarrow V$ be a linear map between $\mathbb{C}$-vector spaces, and let $M=[\widehat{W} \stackrel{u}{\rightarrow} V]$ be the induced 1-motive where $\widehat{W}$ is the formal completion at the origin $(c f .[6,5.2 .5])$. Note that all connected 1-motives are obtained in this way (see 1.3.2). For any embedding $V \subsetneq V^{\prime}$ of vector spaces, we obtain another 1-motive $M^{\prime}=\left[\widehat{W} \stackrel{u^{\prime}}{\rightarrow} V^{\prime}\right]$ such that $M \subsetneq M^{\prime}$. For both $M$ and $M^{\prime}$ then $T_{\phi}(\widehat{W})$ is the infinitesimal group $\widehat{W}, \operatorname{Ker}(u)=\operatorname{Ker}\left(u^{\prime}\right)$ and we cannot distinguish $M$ by $M^{\prime}$ out of the formal group only.

2.2.2. Lemma. We have that $T_{\phi}(F)$ is the formal group $F^{0} \times H_{\mathbb{Z}}$ such that $H_{\mathbb{Z}}$ is the above extension of $F_{\text {ét }}$ by $H_{1}\left(G_{\times}\right)$.

Proof. Since formal groups are closed under extensions $(c f .[6,4.3 .1]) T_{\oint}(F)$ is a formal group, i.e., it is, by construction, an extension of $F$ by $H_{1}(G)$. Observe that (7) yields Lie $(G)$ as the pullback of $\operatorname{Lie}\left(G_{\times}\right)$along exp and $H_{1}(G) \cong H_{1}\left(G_{\times}\right)$. We then get a natural identification of $H_{\mathbb{Z}}$ with the étale part of $T_{\oint}(F)$, i.e., with the pullback of $F_{\text {ét }} \hookrightarrow F$ along $T_{\oint}(F) \rightarrow F$. 
2.2.3. Lemma. If $\sigma:=\bar{\tau}^{-1}: H_{\mathbb{C}} / F_{\text {Hodge }}^{0} \stackrel{\simeq}{\rightarrow}$ Lie $\left(G_{\times}\right)$is the isomorphism induced by (11) then $\sigma$ restricts to $W_{-2}\left(H_{\mathbb{C}}\right) \cong \operatorname{Lie}(T)$ and the following

$$
\begin{array}{ccc}
H_{\mathbb{Z}} & \stackrel{v_{\mathbb{Z}}}{\longrightarrow} & \operatorname{Lie}(G) \\
c \downarrow & & \downarrow p r \\
H_{\mathbb{C}} / F_{\text {Hodge }}^{0} & \stackrel{\sigma}{\longrightarrow} & \operatorname{Lie}\left(G_{\times}\right)
\end{array}
$$

commutes (here $v_{\mathbb{Z}}$ is the restriction of $v$ and $c$ is the canonical map cf. (1)).

Proof. Note that $c=\bar{\tau} \circ \bar{v}_{\mathbb{Z}}$ in (11) and $\bar{v}_{\mathbb{Z}}=$ prov $v_{\mathbb{Z}}$ by Lemma 2.2.2.

2.2.4. Definition. Denote $T_{\oint}(M)$ the formal Hodge structure $(H, V) \in \mathrm{FHS}_{1}^{\mathrm{fr}}$ where

(i) $H:=T_{\oint}(F)=F^{0} \times H_{\mathbb{Z}}, H_{\text {ét }}:=T_{\text {Hodge }}\left(M_{\text {ét }}\right)$, granted by Lemma 2.2.2,

(ii) $V:=\operatorname{Lie}(G), V^{1}:=\operatorname{Lie}(T)+V(G)$ and $V^{0}:=V(G)$,

(iii) the map $v: T_{\phi}(F) \rightarrow \operatorname{Lie}(G)$ defined above, and

(iv) the isomorphism $\sigma:=\bar{\tau}^{-1}: H_{\mathbb{C}} / F_{\text {Hodge }}^{0} \stackrel{\simeq}{\rightarrow} \operatorname{Lie}\left(G_{\times}\right)$grants (1) by Lemma 2.2.3.

We then have $T_{\oint}(M)_{\text {ét }}=T_{\text {Hodge }}\left(M_{\text {ét }}\right) \in \mathrm{MHS}_{1}^{\mathrm{fr}}$ and the construction is clearly functorial (since the diagram (11) is natural) providing a functor

$$
T_{\oint}: \mathcal{M}_{1}^{a, \mathrm{fr}} \longrightarrow \mathrm{FHS}_{1}^{\mathrm{fr}}
$$

such that $T_{\oint}(M)=T_{\text {Hodge }}(M)$ if $M$ is étale (via 1.2.2) and $T_{\oint}(M)=M$ if $M$ is connected.

2.2.5. Remark. Note that by applying $T_{\oint}$ to (9) we get (4), the extension (8) yields (5) and $M$ is special $\Longleftrightarrow T_{\oint}(M)$ is special.

2.3. Conclusion. Summarizing up, see also 1.2 and 1.4, the theorem is proven, e.g., in order to show that $T_{\oint}$ yields an equivalence of categories we can argue as in $[5$, 10.1.3]. For $(H, V) \in \mathrm{FHS}_{1}^{\mathrm{fr}}$ we have constructed, in 1.4, a 1-motive

$$
\overrightarrow{(H, V)}:=\left[H^{0} \times \operatorname{gr}_{0}^{W}\left(H_{\mathbb{Z}}\right) \rightarrow V / W_{-1}\left(H_{\mathbb{Z}}\right)\right]
$$

It is clear that $T_{\oint}(\overrightarrow{(H, V)}) \cong(H, V)$, see $(6)$, which is natural in $(H, V)$. Conversely, for $M=[F \rightarrow G]$ we have $T_{\oint}(M):=\left(T_{\oint}(F)\right.$, Lie $\left.(G)\right)$ such that $\overrightarrow{T_{\oint}(M)} \cong M$ functorially in $M$ by construction. One obtains a duality on $\mathrm{FHS}_{1}^{\text {fr }}$ after Cartier duality on $\mathcal{M}_{1}^{a, \text { fr }}$ by defining

$$
T_{\oint}(M)^{\vee}:=T_{\phi}\left(M^{\vee}\right)
$$

The lemmas 1.2.2 and 2.1.2 further explain the diagram of the main theorem and the remaining claims.

2.3.1. Example. (cf. 2.1.4) For $X$ proper over $\mathbb{C}$ and $M=\left[0 \rightarrow \operatorname{Pic}_{X / \mathbb{C}}^{0}\right]$ we have $T_{\oint}(M)=\left(H^{1}\left(X_{\text {an }}, \mathbb{Z}(1)\right), H^{1}\left(X, \mathcal{O}_{X}\right)\right)$. Here we have $M_{\text {ét }}=\left[0 \rightarrow \mathbb{P i c}_{X . / \mathbb{C}}^{0}\right]$ and a projection

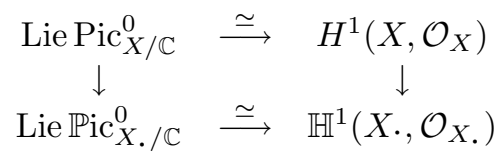


with kernel the additive factor of $\operatorname{Pic}_{X / \mathbb{C}}^{0}$. Further considering $M_{\text {ét }}^{\natural}=\left[0 \rightarrow \mathbb{P i c}_{X . / \mathbb{C}}^{\natural, 0}\right]$ and $T_{\oint}\left(M_{\text {ét }}^{\natural}\right)=\left(H^{1}\left(X_{\text {an }}, \mathbb{Z}(1)\right), H_{D R}^{1}(X)\right)$ we get the extension

$$
0 \rightarrow F_{\text {Hodge }}^{0} \rightarrow T_{\oint}\left(M_{\text {ét }}^{\natural}\right) \rightarrow T_{\text {Hodge }}\left(M_{\text {ét }}\right) \rightarrow 0
$$

2.3.2. Remark. Note that in $2.3 .1 M$ is special but the dual $M^{\vee}$ is not special! Another more striking example is given by taking an abelian variety $X$ and looking at the special 1-motive $\left[0 \rightarrow \operatorname{Pic}_{X / \mathbb{C}}^{\natural, 0}\right]$ which is the universal extension of the dual of $X$. The Cartier dual

$$
\left[0 \rightarrow \operatorname{Pic}_{X / \mathbb{C}}^{\natural, 0}\right]^{\vee}=[\widehat{X} \rightarrow X]
$$

is not special. Actually, in general, the Cartier dual of a connected 1-motive is connected and the dual of étale is étale but the Cartier dual of $M$ special just fits in an extension

dual to (8).

$$
0 \rightarrow M_{\text {ét }}^{\vee} \rightarrow M^{\vee} \rightarrow\left(M^{0}\right)^{\vee} \rightarrow 0
$$

\section{References}

[1] L. Barbieri-Viale, On the theory of 1-motives, in "Algebraic Cycles and Motives" London Mathematical Society Lecture Note Series, Vol. 343, Cambridge University Press, London, 2007. (Proceedings of the workshop on the occasion of the $75^{t h}$ birthday of J.P. Murre - 2004, Lorentz Center, Leiden). Preprint http://arxiv.org/abs/math.AG/0502476

[2] L. Barbieri-Viale and A. Bertapelle, Sharp De Rham realization, Preprint http://arxiv.org/abs/math.AG/0607115

[3] L. Barbieri-Viale, A. Rosenschon and M. Saito, Deligne's conjecture on 1-motives, Annals of Math.(2) 158 no. 2, (2003), 593-633.

[4] S. Bloch and V. Srinivas, Enriched Hodge Structures, in "Algebra, arithmetic and geometry. Part I, II." Papers from the International Colloquium held in Mumbai, January 4-12, 2000. Edited by R. Parimala. Tata Institute of Fundamental Research Studies in Mathematics, Vol. 16, 171-184.

[5] P. Deligne, Théorie de Hodge III, Inst. Hautes Études Sci. Publ. Math 44 (1974), 5-78.

[6] G. Laumon, Transformation de Fourier generalisee, http://arxiv.org/abs/alg-geom/9603004 Preprint IHES (Transformation de Fourier geometrique, IHES/85/M/52) 47 pages.

Università degli Studi di Padova, Dipartimento di Matematica Pura e Applicata, Via Trieste 63, PAdova - I-35121, ItAly

E-mail address: barbieri@math.unipd.it 